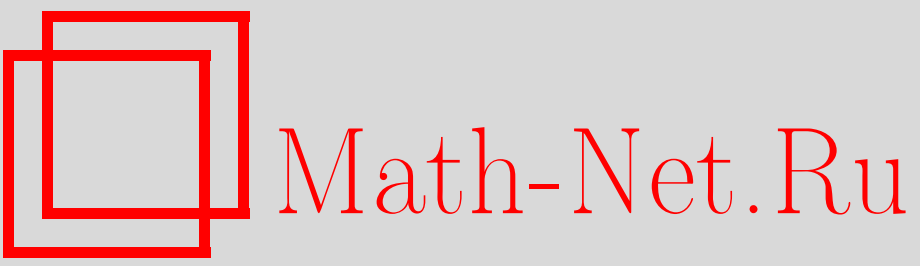

В. С. Владимиров, О нелинейном уравнении $p$-адической открытой струны для скалярного поля, УМН, 2005, том 60, выпуск 6, 73-88

DOI: https://doi.org/10.4213/rm1677

Использование Общероссийского математического портала Math-Net.Ru подразумевает, что вы прочитали и согласны с пользовательским соглашением

http://www . mathnet.ru/rus/agreement

Параметры загрузки:

IP : 54.237 .206 .68

26 апреля 2023 г., 14:13:01 


\title{
О НЕЛИНЕЙНОМ УРАВНЕНИИ $p$-АДИЧЕСКОЙ ОТКРЫТОЙ СТРУНЫ ДЛЯ СКАЛЯРНОГО ПОЛЯ
}

\author{
В. С. ВлАдимиРов
}

\begin{abstract}
Дается обзор резултатов о структуре решений одномерного нелинейного псевдодифференциального уравнения, описывающего динамику (роллинг) $p$-адической открытой струны для скалярного поля тахионов. Указывается на возможность появления разрывных решений при $p=2$. Применяются метод разложения решения в ряды по полиномам Эрмита и метод сведения к нелинейной краевой задаче для уравнения теплопроводности. Приведены некоторые нерешенные вопросы, в частности вопрос о существовании решения краевой задачи при $p=2$.

Библиография: 20 названий.
\end{abstract}

\section{СОДЕРЖАНИЕ}

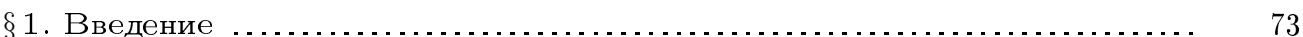

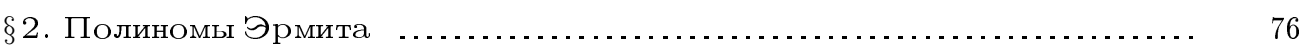

$\S 3$. Свойства оператора $K$.................................... 77

$\S 4$. Разложение решений в ряды по полиномам Эрмита $\ldots \ldots \ldots \ldots \ldots \ldots \ldots . . \ldots 78$

$\S 5$. Связь с решениями уравнения теплопроводности $\ldots \ldots \ldots \ldots \ldots \ldots \ldots \ldots . . \ldots . \ldots . \ldots$

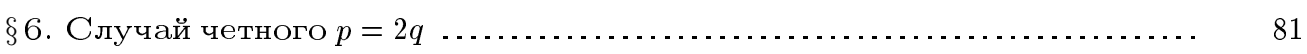

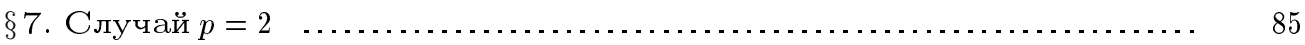

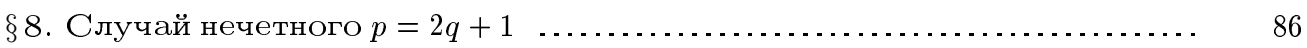

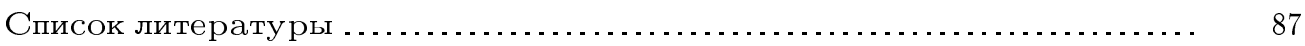

\section{$\S$ 1. Введение}

Динамика открытой $p$-адической струны для скалярного поля тахионов описывается нелинейным псевдодифференциальным уравнением движения вида (см. работы [1]-[14] и литературу в них)

$$
p^{\frac{1}{2} \square} \Phi=\Phi^{p},
$$

где $\Phi=\Phi\left(x_{0}, x_{1}, \ldots, x_{d-1}\right)-$ поле тахионов и

$$
\square=\partial_{t}^{2}-\Delta, \quad \Delta=\partial_{x_{1}}^{2}+\cdots+\partial_{x_{d-1}}^{2}, \quad t=x_{0}
$$

Работа выполнена при частичной финансовой поддержке гранта Президента РФ № НШ-1542. 2003.1 . 
- оператор Даламбера и $p$ - простое число, $p=2,3,5, \ldots$ Будем считать $p$ любым целым числом $\geqslant 2$. Физический смысл имеют вещественные решения уравнения (1.1), и впредь только такие решения будут рассматриваться.

Исследование этого нового класса уравнений (а также более обших уравнений) представляет значительньй интерес не только для $p$-адической математической физики, но и для космологии [7]. В струнной теории поля взаимодействие нелокально [1], что существенно отличает ее от классической локальной теории поля. Этими задачами занимались многие физики и математики с широким использованием компютерной техники: М.Б. Грин, Дж.Г. Шварц, Е. Виттен [1], Л. Брекке, П.Ж. Фрёйнд, М. Олсон, Е. Виттен [2], П. Г. Фрэмптон, Ю. Окада [3], А. Сен [7], Д. Гхошал, А. Сен [8], Н. Мёллер, Б. Цвибах [6], Дж. А. Минахан [12], Н. Мёллер, М. Шнабль [13], Н. Барнаби [14] и др.; в России - И. В. Волович [10], В. С. Владимиров, И. В. Волович и Е. И. Зеленов [4], И.Я. Арефьева, Л.В. Жуковская и А. С. Кошелев [9]. Работы В. С. Владимирова и Я.И. Воловича [11], [15], [16] и Л.В. Жуковской [17] посвяшены чисто математическим и вычислительным аспектам, и этот обзорный доклад, основанньй на них, имеет целью привлечь внимание математиков к новым нелокальным задачам.

В одномерном случае $(d=1)$ с помошью замены неизвестной функции

$$
\varphi(t)=\Phi(t \sqrt{2 \ln p})
$$

уравнение (1.1) приводится к эквивалентному виду

$$
e^{\frac{1}{2} \partial_{t}^{2}} \varphi=\varphi^{p}
$$

Уравнение (1.2) есть нелинейное интегральное уравнение вида [15]

$$
\frac{1}{\sqrt{\pi}} \int_{-\infty}^{\infty} e^{-(t-\tau)^{2}} \varphi(\tau) d \tau=\varphi^{p}(t), \quad t \in \mathbb{R} .
$$

Исследование уравнения (1.3) - задача классического анализа: от $p$-адических чисел здесь осталось только целое число $p$, к тому же оно не обязано быть простым.

Решение уравнения (1.3) предполагается в классе измеримых функций $\varphi(t)$, удовлетворяюших условию роста

$$
|\varphi(t)| \leqslant C \exp \left[(1-\varepsilon) t^{2}\right] \text { при любом } \varepsilon>0, \quad t \in \mathbb{R} .
$$

Физический смысл имеют следуюшие краевые задачи для уравнения (1.3):

$$
\begin{aligned}
& \lim _{t \rightarrow-\infty} \varphi(t)=0, \quad \lim _{t \rightarrow \infty} \varphi(t)=1, \quad p \text { - четное; } \\
& \lim _{t \rightarrow-\infty} \varphi(t)=-1, \quad \lim _{t \rightarrow \infty} \varphi(t)=1, \quad p \text { - нечетное. }
\end{aligned}
$$

Наряду с решением $\varphi(t)$ уравнения (1.3), функции $\varphi(-t)$ и $\varphi\left(t+t_{0}\right)$ при любом $t_{0}$, а при нечетном $p$ и функция $-\varphi(t)$ также являются решением его. Поэтому решение уравнения (1.3) неединственно, если оно сушествует.

Тем не менее справедлива следуюшая теорема сушествования (и единственности) (при $\alpha=0$ она доказана в [15]). 
ТеОремА. Все вещественные решения уравнения (1.3) вида

$$
\varphi(t)=e^{\alpha t^{2}} \psi(t), \quad \psi \in \widetilde{S_{+}^{\prime}}, \quad \alpha \in \mathbb{R},
$$

где $\widetilde{S_{+}^{\prime}}-$ алгебра по умножению преобразований Фурье обобщенных функчий медленного роста с носителем на полуоси $[0, \infty)$, представляются с виде

$$
\varphi(t)= \begin{cases}0, \pm 1, \pm \varphi_{0}(t), & p-\text { нечетное } \\ 0,1, \varphi_{0}(t), & p-\text { четное }\end{cases}
$$

əде

$$
\varphi_{0}(t)=p^{\frac{1}{2(p-1)}} \exp \left(\frac{p-1}{p} t^{2}\right)
$$

Для исследования и решения интегрального уравнения (1.3) и соответствуюших краевых задач рассматриваются:

- метод разложения по полиномам Эрмита;

- метод сведения к нелинейной краевой задаче для уравнени теплопроводности;

- нерешенные вопросы.

Нам понадобится шкала весовых сепарабельных гильбертовых пространств $L_{2}^{\alpha}$, $0<\alpha<\infty$, состоящих из измеримых квадратично-суммируемых функций на $\mathbb{R}$ по мepe

$$
d \mu_{\alpha}(t)=\sqrt{\frac{\alpha}{\pi}} e^{-\alpha t^{2}} d t, \quad \int_{-\infty}^{\infty} d \mu_{\alpha}(t)=1
$$

со следуюшими скалярньм произведением и нормой

$$
(f, g)_{\alpha}=\int_{-\infty}^{\infty} f(t) \bar{g}(t) d \mu_{\alpha}(t), \quad\|f\|_{\alpha}=\sqrt{(f, f)_{\alpha}}, \quad f, g \in L_{2}^{\alpha}, \quad \alpha>0 .
$$

Вложение $L_{2}^{\alpha} \subset L_{2}^{\beta}, \alpha<\beta$, плотно и непрерьвно.

Справедливы следующие известные утверждения [15], [16].

УТВЕРЖДЕНИЯ. Пусть $\varphi-$ решение уравнения (1.3). Тогда

1) если $\varphi(t)$ - ограничено, то оно кусочно-вещественно-аналитично (при р нечетном - непрерывно) и справедливо неравенство

$$
|\varphi(t)|<1, \quad t \in \mathbb{R}
$$

2) ecлu

$$
\lim _{t \rightarrow \infty} \varphi(t)=a, \quad|a|<\infty,
$$

то $а=0$ или $a=1$ при р четном и $a=0$ или $a= \pm 1$ при р нечетном; при әтом

$$
\lim _{t \rightarrow \infty}\left(\varphi^{p}\right)^{\prime}(t)=0
$$

и если $a \neq 0$, mo $u \lim _{t \rightarrow \infty} \varphi^{(n)}(t)=0, n=1,2, \ldots$. 


\section{§. Полиномы Эрмита}

Полиномами Эрмита называются полиномы вида [18]

$$
H_{n}(x)=(-1)^{n} e^{x^{2}} \frac{d^{n}}{d x^{n}} e^{-x^{2}}, \quad n=0,1,2, \ldots,
$$

так что $H_{0}(x)=1, H_{1}(x)=2 x, H_{2}(x)=4 x^{2}-2, H_{3}(x)=8 x^{3}-12 x, \ldots$.

Они образуют полную ортогональную систему в гильбертовом пространстве $L_{2}^{1}$, причем

$$
\|H\|_{1}^{2}=\int_{-\infty}^{\infty} H_{n}^{2}(x) d \mu_{1}(x)=2^{n} n !
$$

так что $f \in L_{2}^{1}$ разлагается в ряд

$$
f(x)=\sum_{n=0}^{\infty}\left(f, H_{n}\right)_{1} \frac{H_{n}(x)}{2^{n} n !} \text { в } \quad L_{2}^{1}
$$

Имеют место следующие равенства [19]:

$$
\left(x^{m}, H_{n}\right)_{1}= \begin{cases}2^{n-m} m !\left(\frac{m-n}{2} !\right)^{-1}, & m \geqslant n \text { и } n \equiv m, \\ 0, & m<n \text { или } n \neq m .\end{cases}
$$

Здесь принято обозначение: $a \equiv b$ или $a \not \equiv b$, если целые числа $a$ и $b$ одной или разной четности соответственно.

Разложение полиномов Эрмита по степеням х [20]

$$
H_{n}(x)=n ! \sum_{m=0, m \equiv n}^{n} c_{n, m} x^{m}, \quad n=0,1,2, \ldots,
$$

где

$$
c_{n, m}= \begin{cases}(-1)^{\frac{n-m}{2}} \frac{2^{m}}{m !}\left(\frac{m-n}{2} !\right)^{-1}, & m \equiv n \text { и } m \leqslant n, \\ 0, & m \neq n \text { или } m>n .\end{cases}
$$

Модифицированные полиномы Эрмита-это полиномы вида [16]

$$
V_{n}(x)=2^{-n / 2} H_{n}\left(\frac{x}{\sqrt{2}}\right), \quad n=0,1,2, \ldots,
$$

так что $V_{0}(x)=1, V_{1}(x)=x, V_{2}(x)=x^{2}-1, V_{3}(x)=x^{3}-3 x, \ldots$

Они образуют полную ортогональную систему в гильбертовом пространстве $L_{2}^{1 / 2}$, причем

$$
\left\|V_{n}\right\|_{1 / 2}^{2}=\int_{-\infty}^{\infty} V_{n}^{2}(x) d \mu_{1 / 2}(x)=n !
$$


так что $f \in L_{2}^{1 / 2}$ разлагается в ряд

$$
f(x)=\sum_{n=0}^{\infty}\left(f, V_{n}\right)_{1 / 2} \frac{V_{n}(x)}{n !} \text { в } L_{2}^{1 / 2} .
$$

Имеют место следуюшие равенства [20]

$$
\begin{aligned}
\left(H_{m}, V_{n}\right)_{1 / 2} & = \begin{cases}2^{n} m !\left(\frac{m-n}{2} !\right)^{-1}, & m \geqslant n \text { и } n \equiv m, \\
0, & m<n \text { или } n \neq m ;\end{cases} \\
\left(H_{n}, V_{m}\right)_{1} & = \begin{cases}(-1)^{\frac{m-n}{2}} 2^{n-m} m !\left(\frac{m-n}{2} !\right)^{-1}, & m \geqslant n \text { и } n \equiv m, \\
0, & m<n \text { или } n \neq m .\end{cases}
\end{aligned}
$$

Разложсение полиномов $V_{n}$ по $H_{n}$ и полиномов $H_{n}$ по $V_{n}$ следует из формул $(2.3),(2.9),(2.10)$ и $(2.11)$ :

$$
\begin{aligned}
& V_{n}(x)=2^{-n} n ! \sum_{m=0, m \equiv n}^{n}(-1)^{\frac{n-m}{2}}\left(\frac{m-n}{2} !\right)^{-1} \frac{H_{m}(x)}{m !}, \\
& H_{n}(x)=n ! \sum_{m=0, m \equiv n}^{n} \frac{2^{m}}{m !}\left(\frac{m-n}{2} !\right)^{-1} V_{m}(x) .
\end{aligned}
$$

Интегральное представление для модифицированных полиномов Эрмита [20]

$$
V_{n}(x)=\sqrt{\frac{2}{\pi}} \int_{-\infty}^{\infty} H_{n}(\tau) e^{-2(t / 2-\tau)^{2}} d \tau, \quad n=0,1,2, \ldots
$$

Пусть $f \in L_{2}^{1 / 2}$. Тогда в силу (1.2) $f \in L_{2}^{1}$ и справедливы равенства

$$
\sum_{n=0}^{\infty} a_{n} \frac{H_{n}(x)}{2^{n} n !}=f(x)=\sum_{n=0}^{\infty} b_{n} \frac{V_{n}(x)}{n !} \text { в } L_{2}^{1},
$$

причем коэффициенты $a_{n}=\left(f, H_{n}\right)_{1}$ и $b_{n}=\left(f, V_{n}\right)_{1 / 2}$ выражаются один через другой по формулам

$$
\begin{aligned}
& a_{n}=\sum_{m \geqslant n, m \equiv n}(-1)^{\frac{m-n}{2}} 2^{n-m}\left(\frac{m-n}{2} !\right)^{-1} b_{m}, \quad n=0,1, \ldots, \\
& b_{n}=\sum_{m \geqslant n, m \equiv n} 2^{n-m}\left(\frac{m-n}{2} !\right)^{-1} a_{m}, \quad n=0,1,2, \ldots
\end{aligned}
$$

\section{$\S$ 3. Свойства оператора $K$}

Обозначим через $K$ линейный интегральный оператор в уравнении (1.3)

$$
\varphi \rightarrow(K \varphi)(t) \equiv \frac{1}{\sqrt{\pi}} \int_{-\infty}^{\infty} e^{-(t-\tau)^{2}} \varphi(\tau) d \tau .
$$

Имеют место следуюшие леммы [16].

Лемма 1. Оператор $K$ переводит всякую функиию $f(t)$, удовлетворяющую условию (1.4), в целую функиию $(K f)(z)$ с оценкой

$$
|(K f)(z)| \leqslant \frac{C}{\sqrt{\varepsilon}} \exp \left[y^{2}+(1 / \varepsilon-1) t^{2}\right], \quad z=t+i y .
$$


Лемма 2. Oператор $K$ переводит функиию $f \in L_{2}^{\alpha}, 0<\alpha<2$ в целую функцию $(K f)(z)$ с оченкой

$$
|(K f)(z)| \leqslant\|f\|_{\alpha}(2-\alpha)^{-1 / 4} \exp \left(y^{2}+\frac{\alpha}{2-\alpha} t^{2}\right), \quad z=t+i y .
$$

Лемма 3. Оператор $K$ ограничен из $L_{2}^{\alpha}, 0<\alpha<2$, в $L_{2}^{\beta}, \beta>\frac{2 \alpha}{2-\alpha}$, и справедлива оценка

$$
\|K f\|_{\beta} \leqslant\left(\frac{2 \alpha-2 \alpha^{2}}{\beta-\alpha^{2}}\right)^{-1 / 4}\|f\|_{\alpha}, \quad f \in L_{2}^{\alpha}
$$

Лемма 4. Если $f \in L_{2}^{1}$, то ее образ $(K f)(t)$ разлагается в ряд Тейлора

$$
(K f)(t)=\sum_{n=0}^{\infty} a_{n} \frac{t^{n}}{n !}, \quad a_{n}=\left(f, H_{n}\right)_{1},
$$

сходящийся равномерно на каждом компакте $\mathbb{R}$. Если $f \in L_{2}^{1 / 2}$, то

$$
(K f)(t)=\sum_{n=0}^{\infty} b_{n} \frac{H_{n}(t)}{2^{n} n !} \quad \text { e } \quad L_{2}^{1}, \quad b_{n}=\left(K f, H_{n}\right)_{1},
$$

при этом справедливы равенства

$$
\left(K f, H_{n}\right)_{1}=\left(f, V_{n}\right)_{1 / 2}, \quad n=0,1,2, \ldots
$$

\section{§4. Разложение решений в ряды по полиномам Эрмита}

Пусть $\varphi$ есть решение уравнения (1.3) из $L_{2}^{1}$, так что $\varphi^{p}=K \varphi$. Тогда, обозначая $a_{n}=\left(\varphi, H_{n}\right)_{1}$, в силу $(2.3)$ будем иметь

$$
\varphi(t)=\sum_{n=0}^{\infty} a_{n} \frac{H_{n}(t)}{2^{n} n !} \quad \text { в } \quad L_{2}^{1}, \quad \sum_{n=0}^{\infty} \frac{a_{n}^{2}}{2^{n} n !}=\|\varphi\|^{2} .
$$

При этом функция $\varphi^{p}(t)$ есть след целой функции $A(z)=(K \varphi)(z)$, удовлетворяющей оценке (3.2) при $\alpha=1$ :

$$
|A(z)| \leqslant\|\varphi\|_{1} e^{|z|^{2}}, \quad z \in \mathbb{C},
$$

и по лемме 4 она разлагается в ряд Тейлора (3.4) [16]

$$
\varphi^{p}(t)=\sum_{n=0}^{\infty} a_{n} \frac{t^{n}}{n !}
$$

Если же решение уравнения (1.3) $\varphi \in L_{2}^{1 / 2}$, то по лемме $3 \varphi^{p} \in L_{2}^{1}$ и справедливо неравенство

$$
\left\|\varphi^{p}\right\|_{1} \leqslant \sqrt{2}\|\varphi\|_{1 / 2}
$$


а по лемме 4 справедливы равенства (3.6) (интегральные законы сохранения):

$$
\left(\varphi^{p}, H_{n}\right)_{1}=b_{n}=\left(\varphi, V_{n}\right)_{1 / 2}, \quad n=0,1,2, \ldots
$$

Поэтому функция $\varphi^{p}(t)$ разлагается в ряд по полиномам Эрмита, а функция $\varphi(t)$ - по модифицированным полиномам Эрмита:

$$
\begin{aligned}
\varphi^{p}(t) & =\sum_{n=0}^{\infty} b_{n} \frac{H_{n}(t)}{2^{n} n !} \text { в } L_{2}^{1}, \\
\varphi(t) & =\sum_{n=0}^{\infty} b_{n} \frac{V_{n}(t)}{n !} \text { в } L_{2}^{1 / 2} .
\end{aligned}
$$

\section{$\S$ 5. Связь с решениями уравнения теплопроводности}

Интегральное уравнение (1.3) эквивалентно следующей краевой задаче для уравнения теплопроводности [16]:

$$
\begin{aligned}
u_{x} & =\frac{1}{4} u_{t t}, \quad 0<x \leqslant 1, \quad t \in \mathbb{R}, \\
u(0, t) & =\varphi(t), \quad u(1, t)=\varphi^{p}(t), \quad t \in \mathbb{R} .
\end{aligned}
$$

(Обратим внимание, что переменные $x$ и $t$ в (5.1) поменялись местами по сравнению с классическим уравнением теплопроводности.)

Решением краевой задачи (5.1)-(5.2) назовем всякую измеримую функцию $u(x, t)$, ограниченную условием роста (1.4) по $t$, причем такую, что $C$ не зависит от $x$. Функцию $u(x, t)$ назовем интерполирующей между решением $\varphi(t)$ и его степенью $\varphi^{p}(t)$.

Отметим, что интерполирующая функция представляется формулой Пуассона для уравнения (5.1):

$$
u(x, t)=\frac{1}{\sqrt{\pi x}} \int_{-\infty}^{\infty} \varphi(\tau) \exp \left[-\frac{(t-\tau)^{2}}{x}\right], \quad 0<x \leqslant 1 .
$$

Если функция $\varphi$ удовлетворяет условию роста

$$
|\varphi(t)| \leqslant C \exp \left(\varepsilon t^{2}\right) \text { при любом } \varepsilon>0, \quad t \in \mathbb{R},
$$

то формула (5.3) дает ее вещественно-аналитическое продолжение в область $x>1$, $t \in \mathbb{R}$ и, далее, - аналитическое продолжение $u(\zeta, z)$ в комплексную область $T^{+} \times \mathbb{C}$, где $T^{+}$- правая полуплоскость $\operatorname{Re} \zeta=x>0$.

Из представления (5.3) следует: если $\varphi$ есть решение краевых задач (1.3)-(1.5) или (1.3)-(1.6), то $|u(x, t)|<1$ при $0 \leqslant x, t \in \mathbb{R}, u(x, t)$ удовлетворяет краевым условиям (1.5) или (1.6) соответственно и $u(x, t)>0$ при $x>1$ для задачи (1.3)-(1.5).

ПримеР. Для решения $\varphi_{0}(t)($ см. (1.8)) уравнения (1.3) интерполируюшая функция равна

$$
u(x, t)=p^{\frac{1}{2(p-1)}}(1-x+x / p)^{-\frac{1}{2}} \exp \left[\frac{t^{2}(p-1)}{p-x p+x}\right] .
$$


ТЕОРема [16]. Пусть $и(x, t)$ - интерполирующая функиия междуу решением $\varphi$ краевых задач (1.3)-(1.5) и (1.3)-(1.6) и его степенью $\varphi^{p}$. Тогда справедливо равенство

$$
\int_{-\infty}^{\infty} \varphi^{2}(t)\left[1-\varphi^{2 p-2}(t)\right] d t=\frac{1}{2} \int_{0}^{1} \int_{-\infty}^{\infty} u_{t}^{2}(x, t) d x d t
$$

и законы сохранения

$$
\int_{-\infty}^{\infty}[u(x, t)-\varphi(t)] d t=0=\int_{-\infty}^{\infty}\left[u(x+1, t)-\varphi^{p}(t)\right] d t=0, \quad x \geqslant 0
$$

СлЕДСТВИя. 1) Для того чтобь интеграль

$$
\begin{cases}\int_{-\infty}^{0} \varphi^{2}(t) d t, \quad \int_{0}^{\infty}\left[1-\varphi^{2 p-2}(t)\right] d t, & \text { для задачи (1.3)-(1.5) } \\ \int_{-\infty}^{\infty}\left[1-\varphi^{2 p-2}(t)\right] d t, & \text { для задачи (1.3)-(1.6) }\end{cases}
$$

сходились, необходимо и достаточно, чтобь

$$
\int_{0}^{1} \int_{-\infty}^{\infty} u_{t}^{2}(x, t) d x d t<\infty
$$

2) Для задачи (1.3)-(1.5) сходятся интеграль

$$
\int_{0}^{\infty}\left[1-\varphi^{p-1}(t)\right] d t, \quad \int_{0}^{\infty}[1-\varphi(t)] d t, \quad \int_{0}^{\infty}[1-u(x, t)] d t, \quad x \geqslant 1
$$

а если $\varphi(t)$ знакопостоянна при $t<c$, то сходятся и интеграль

$$
\int_{-\infty}^{0} \varphi(t) d t, \quad \int_{-\infty}^{0} u(x, t) d t, \quad x \geqslant 1
$$

3) Для задачи (1.3)-(1.6) сходятся интеграль:

$$
\begin{gathered}
\int_{-\infty}^{\infty}\left[1-\varphi^{p-1}(t)\right] d t, \quad \int_{0}^{\infty}[1-\varphi(t)] d t, \quad \int_{-\infty}^{0}[1+\varphi(t)] d t \\
\int_{0}^{\infty}[1-u(x, t)] d t, \quad \int_{-\infty}^{0}[1+u(x, t)] d t, \quad x \geqslant 0 .
\end{gathered}
$$

Вопрос. Можсно ли избавиться в 2) от условия $\varphi(t)$ знакопостоянства при $t<c$ ? 


\section{§. Случай четного $p=2 q$}

Уравнение (1.3) принимает вид

$$
\varphi^{2 q}(t)=\frac{1}{\sqrt{\pi}} \int_{-\infty}^{\infty} \varphi(\tau) e^{-(t-\tau)^{2}} d \tau, \quad q=1,2, \ldots
$$

В работе [15] получен такой результат: не существует непрерывных неотрицательных решений краевой задачи (6.1), (1.5).

Пусть существует кусочно-непрерывное решение $\varphi(t)$ краевой задачи уравнения $(6.1),(1.5)$. Тогда $\varphi(t)$ удовлетворяет уравнению

$$
\varphi^{2 q}(t)=A(t)
$$

где $A(t) \geqslant 0$ - следцелой функции $A(z)$, удовлетворяюшей оценке (4.2). В окрестности каждой точки сушествуют два вешественных решения уравнения (6.2)

$$
\varphi(t)= \pm A^{\frac{1}{2 q}}(t)
$$

Поэтому глобальная структура решения $\varphi(t)$ зависит от ее точек разрьва I рода $T_{k}$ и от вешественных нулей $t_{k}$ целой функции $A(z)$. Множества $\left\{T_{k}\right\}$ и $\left\{t_{k}\right\}$ ограничены сверху, конечны либо счетны.

В окрестности нуля $t_{k}$ функция $\varphi^{2 q}(t)$ в силу $(6.2)$ имеет представление

$$
\varphi^{2 q}(t)=\frac{a_{2 \sigma_{k}}}{\left(2 \sigma_{k}\right) !}\left(t-t_{k}\right)^{2 \sigma_{k}}\left[1+O\left(\left|t-t_{k}\right|\right)\right]
$$

где $a_{2 \sigma_{k}}>0,2 \sigma_{k}-$ кратность нуля $t_{k}$. При этом справедливы равенства

$$
\frac{2^{2 \sigma_{k}}}{\sqrt{\pi}} \int_{-\infty}^{\infty} \varphi(\tau)\left(t_{k}-\tau\right)^{n} e^{-\left(t_{k}-\tau\right)^{2}} d \tau= \begin{cases}a_{2 \sigma_{k}}>0, & n=2 \sigma_{k} \\ 0, & n=0,1, \ldots, 2 \sigma_{k}-1\end{cases}
$$

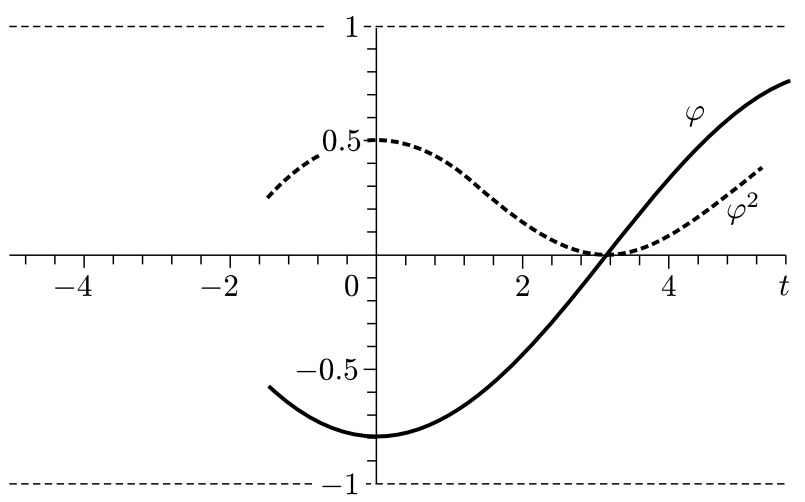

Рис. 1 


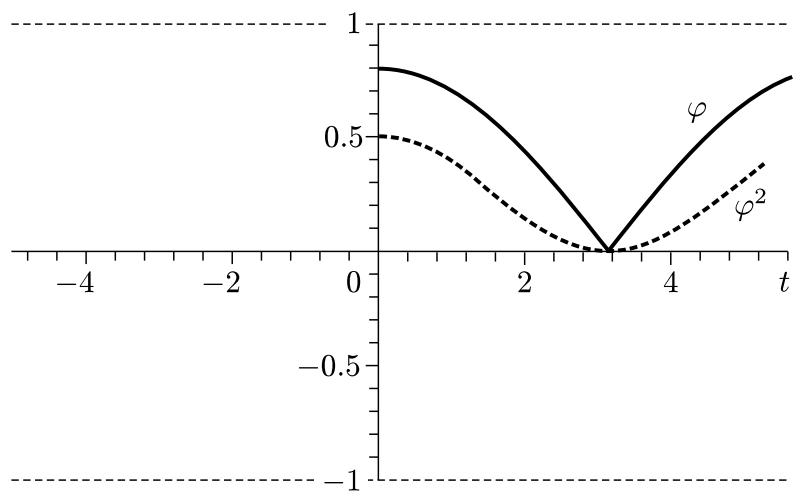

Рис. 2

В работах [15], [16] получены следуюшие результаты.

Теорема. Если $\varphi$ - решение краевой задачи (6.1), (1.5), то:

1) решение $\varphi(t)$ - кусочно-вещественно-аналитическое всюду за исключением его нулей и точек разрыва I рода;

2) если $\varphi^{2 q}(t)$ не имеет нулей или имеет только один нуль, то $\varphi(t)$ может иметь разрыввы I рода;

3) если $\varphi^{2 q}(t)$ имеет только два нуля $t_{0} u t_{1} u \varphi(t)$ непрерьвна, то она имеет вид (см. рис. 3)

$$
\varphi(t)= \begin{cases}A^{\frac{1}{2 q}}(t), & t>0 \\ -A^{\frac{1}{2 q}}(t), & t_{1}<t<0 \\ A^{\frac{1}{2 q}}(t), & t<t_{1}\end{cases}
$$

4) в окрестности нуля $t_{k}$ решение $\varphi(t)$ имеет одно из четырех представлений (см. рис. 1 и 2 ):

$$
\varphi(t)= \pm\left\{\begin{array}{c}
1 \\
\operatorname{sgn} t
\end{array}\right\}\left[\frac{a_{2 \sigma_{k}}}{\left(2 \sigma_{k}\right) !}\right]^{\frac{1}{2 q}}\left|t-t_{k}\right|^{\frac{\sigma_{k}}{q}}\left[1+O\left(\left|t-t_{k}\right|\right)\right]
$$

5) в окрестности точки разрыва I рода $T_{k}$ функция $\varphi(t)$ имеет представление (см. рис. 4 и 5)

$$
\varphi(t)= \pm \operatorname{sgn}\left(t-T_{k}\right) A^{\frac{1}{2 q}}(t), \quad t \neq T_{k}
$$

и ее скачок в точке $T_{k}$, равен

$$
\pm 2 A^{\frac{1}{2 q}}\left(T_{k}+0\right)
$$

6) если решение $\varphi(t)$ непрерьвно, то число перемен знака его не превосходит числа нулей функиии $\varphi^{2 q}(t)$ и не меньше $\sup _{k} 2 \sigma_{k}$. 


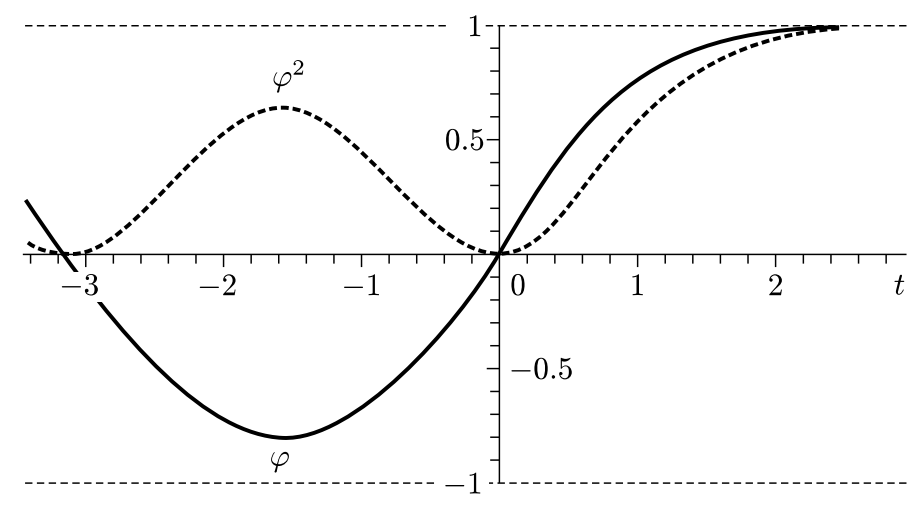

Рис. 3

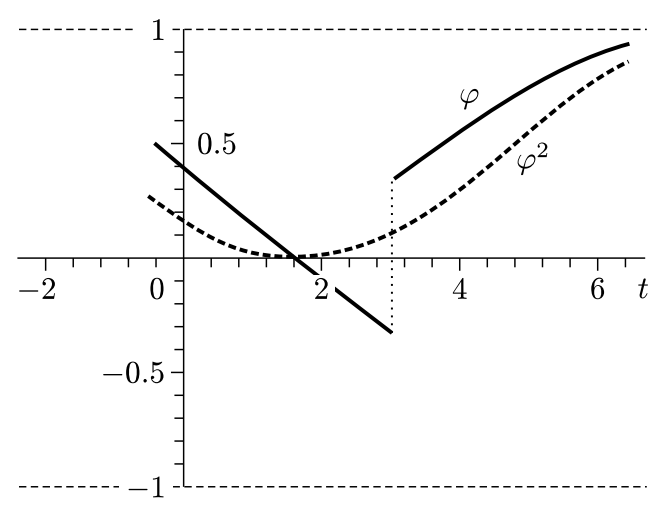

Рис. 4

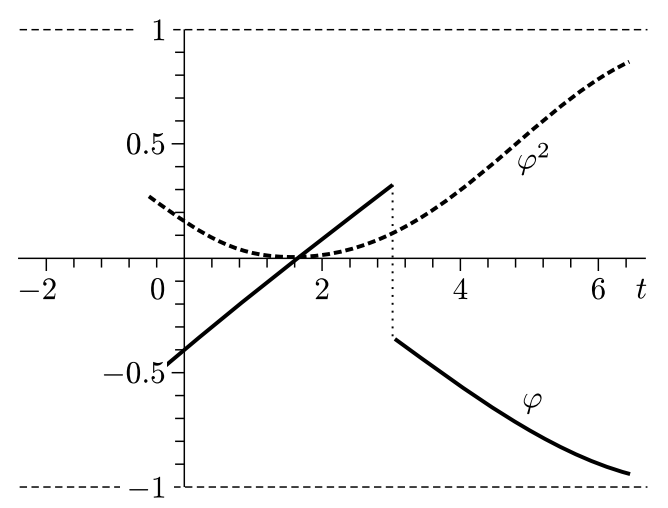

Рис. 5 
С помощью принципа сжатых отображений для нулей интерполирующей функции $u(x, t)$ доказьвается следующая теорема [16].

ТЕОРема (о ветвлении нулей). Пусть $u(x, t)-$ интерполирующая функция между решением $\varphi$ краевой задачи (5.1)-(5.2) и $\varphi^{2 q}$ такая, что и $(1, t)$ имеет в точке $t=0$ нуль кратности $2 n$. Тогда уравнение

$$
u(1-\varepsilon, t)=0 \quad \text { npu } \quad \varepsilon \rightarrow+0
$$

имеет ровно 2 простых вещественных корней

$$
t_{k}^{ \pm}(\varepsilon)=\frac{1}{2} \lambda_{k}^{ \pm} \sqrt{\varepsilon}+O(\varepsilon), \quad k=1, \ldots, n,
$$

где $\lambda_{k}^{ \pm}-$корни универсального уравнения

$$
H_{2 n}\left(\frac{\lambda}{2}\right) \equiv \sum_{m=0}^{n}(-1)^{m} \frac{\lambda^{2 n-2 m}}{(2 n-2 m) ! m !}=0 .
$$

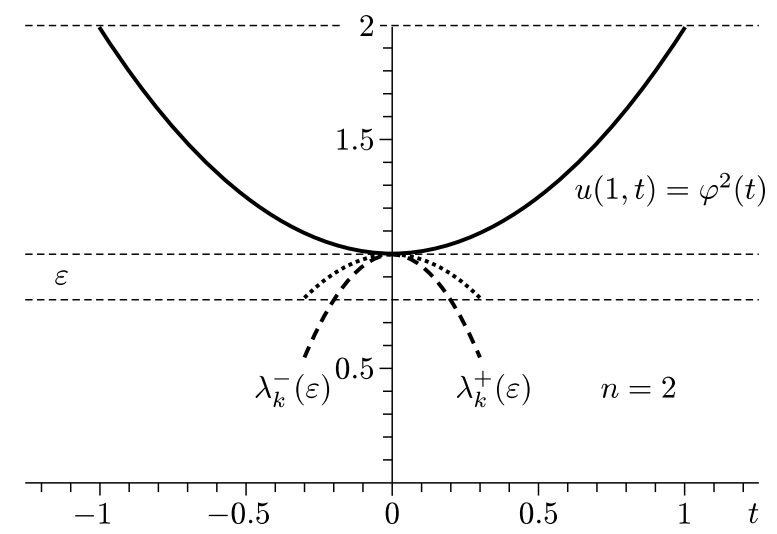

Рис. 6

ПримеРЫ. Уравнение (6.12) и его корни имеют следующий вид: при $n=1 \quad \lambda^{2}=2, \lambda^{ \pm}= \pm \sqrt{2}$; при $n=2 \lambda^{4}-12 \lambda^{2}+12=0$,

$$
\lambda_{1}^{ \pm}= \pm \sqrt{6+2 \sqrt{6}}, \quad \lambda_{2}^{ \pm}= \pm \sqrt{6-2 \sqrt{6}}
$$

при $n=3 \lambda^{6}-30 \lambda^{4}+180 \lambda^{2}-120=0$,

$$
\lambda_{1}^{ \pm} \approx \pm 0.845, \quad \lambda_{2}^{ \pm} \approx \pm 2.67, \quad \lambda_{3}^{ \pm} \approx \pm 4.70
$$

Вопросы. 1) Существует ли непрерывное или разрывное решение краевой задачи (6.1), (5.1)?

2) Всегда ли в нуле функиии $\varphi^{2 q}(t)$ имеет место перемена знака у решения $\varphi(t)$ ? 


\section{$\S$ 7. Случай $p=2$}

Уравнение (1.3) принимает вид

$$
\varphi^{2}(t)=\frac{1}{\sqrt{\pi}} \int_{-\infty}^{\infty} \varphi(\tau) e^{-(t-\tau)^{2}} d \tau
$$

Пусть $\varphi(t)$ - решение уравнения $(7.1)$ из $L_{2}^{1}$. Вьведем формально бесконечную систему нелинейных уравнений для коэффициентов $a_{n}$ в разложении (4.3) функции $\varphi^{2}$ и в разложении (4.1) функции $\varphi$. Ряд Тейлора для $\varphi(t)$ вытекает из ряда (4.1) с помошњю переразложения (2.5) полиномов Эрмита по степеням $t$. Тогда

$$
a_{n}=n ! \sum_{k+i=n}\left(\sum_{m=k, m \equiv k}^{\infty} \frac{a_{m}}{2^{m}} c_{m, k}\right)\left(\sum_{s=i, s \equiv i}^{\infty} \frac{a_{s}}{2^{s}} c_{s, i}\right), \quad n=0,1,2, \ldots
$$

Отметим, что ряды в (7.2) сходятся в силу равенства Парсеваля-Стеклова (4.1) и неравенства Коши-Буняковского.

Анализ нелинейной системы (7.2) показывает [16], что уравнение для коэффициента $a_{n}, n \geqslant 1$, при условии $a_{0}>0$ линейно относительно неизвестных $a_{k}, k \equiv n, k=n, n+$ $1, \ldots$ Аналогичное свойство имеет место и при $a_{0}=a_{1}=0, a_{2}>0$ и т. д.

Рассмотрим усеченную систему (7.2) при $n=0,1,2,3$ (( $n=3)$-приближение), полагая $a_{4}=a_{5}=\cdots=0$. Используем для этого равенства $(2.6)$ для коэффициентов $c_{m, k}$. При $n=0$

$$
a_{0}=\left(\sum_{m=0}^{3}(-1)^{m} \frac{a_{2 m}}{4^{m} m !}\right)^{2} \geqslant 0
$$

Рассмотрим для определенности случай $a_{0}>0$. (Случай $a_{0}=0$ рассматривается аналогично, см. [16].) В результате получим следующую нелинейную систему уравнений $(\varepsilon= \pm)$ для коэффициентов $a_{0}, a_{1}, a_{2}, a_{3}$ :

$$
\begin{aligned}
& a_{0}=\varepsilon \sqrt{a_{0}}+a_{2} / 4, \\
& a_{1}=2 \varepsilon \sqrt{a_{0}}\left(a_{1}-a_{3} / 4\right), \\
& a_{2}=\frac{a_{1}^{2}}{2 a_{0}}+2 \varepsilon \sqrt{a_{0}} a_{2}, \\
& a_{3}=2 \varepsilon \sqrt{a_{0}} a_{3}+\frac{3 a_{1} a_{2}}{\varepsilon \sqrt{a_{0}}} .
\end{aligned}
$$

Рассмотрим линейную систему относительно коэффициентов $a_{1}$ и $a_{3}$, состоящую из уравнений $\left(7.4_{1}\right)$ и $\left(7.4_{3}\right)$ :

$$
\left\{\begin{array}{c}
\left(1-\varepsilon \sqrt{a_{0}}\right) a_{1}+1 / 2 \varepsilon \sqrt{a_{0}} a_{3}=0 \\
-3 \frac{a_{2} a_{1}}{\varepsilon \sqrt{a_{0}}}+\left(1-2 \varepsilon \sqrt{a_{0}}\right) a_{3}=0
\end{array}\right.
$$

Определитель этой системы равен

$$
D=1+10 a_{0}-10 \varepsilon \sqrt{a_{0}} .
$$


Для решений системы (7.4) справедливо следуюшее утверждение.

УТВЕРЖДЕНИЕ. Если $a_{0}>0$ u $D \neq 0, m o$

а) $a_{0}=1$ и $a_{1}=a_{2}=a_{3}=0$, что соответствует тривиальному решению $\varphi(t)=1$

b) $a_{0}=1 / 4, a_{1}=0, a_{2}=-1, a_{3}=0$ ( и тогда $\left.\varepsilon=+\right)$, что соответствует приближсенному решению

$$
\varphi_{1}(t) \approx \frac{1}{2}\left(1-t^{2}\right) .
$$

Если $a_{0}>0$ и $D=0$ ( и тогда $\left.\varepsilon=+\right)$, mо

c) $a_{0}=0.4000+\sqrt{0.15} \approx 0.7873, a_{1} \approx \pm 0.6984, a_{2}=-0.4000, a_{3} \approx \pm 1.219$, что соответствует двум приближенныц решениям

$$
\begin{aligned}
\varphi_{2}(t) & \approx 0.7873 \pm 0.6984 t-0.05000 H_{2}(t) \pm 0.02540 H_{3}(t) \\
& =0.8873 \pm 0.3936 t-0.2000 t^{2} \pm 0.2032 t^{3} .
\end{aligned}
$$

Вопрос. Являются ли приближенные решения (7.7) и (7.8) "паразитныци" или это первые члены неизвестных решений из $L_{2}^{1}$ ?

По крайней мере до $t^{4}$ квадраты этих решений совпадают с их квадратами, вычисленными по формуле (4.3):

$$
\begin{aligned}
\varphi_{1}^{2} & \approx \frac{1}{4}\left(1-2 t^{2}\right), \\
\varphi_{2}^{2}(t) & \approx 0.7873 \pm 0.6984 t-0.2000 t^{2} \pm 0.2032 t^{3} .
\end{aligned}
$$

Изложенньй метод для решения у равнения (7.1) применяется и к решению $\varphi(t)$ краевой задачи $(7.1),(1.5)[16]$. Это решение ищется в виде

$$
\varphi(t)=\frac{1}{2}+\frac{1}{2} \operatorname{erf}(t)+e^{-\left(\alpha^{2}-1\right) t^{2}} \sum_{m=0}^{\infty} c_{m} H_{m}(\alpha t)
$$

с неизвестными коэффициентами $c_{0}, c_{1}, c_{2}, \ldots$, где $\alpha>1$ - некоторый параметр.

\section{§ 8. Случай нечетного $p=2 q+1$}

Совсем другая картина наблюдается при нечетных $p$. Уравнение (1.3) принимает вид

$$
\varphi^{2 q+1}(t)=\frac{1}{\sqrt{\pi}} \int_{-\infty}^{\infty} \varphi(\tau) e^{-(t-\tau)^{2}} d \tau, \quad q=1,2, \ldots
$$

Для построения решений уравнения (8.1) можно применить метод разложения по полиномам Эрмита, изложенньй в $§ 7$. Однако вычисления будут довольно громоздкими 
даже в случае $p=3$. Наконец, для решения краевой задачи (8.1), (1.6) может оказаться полезной подстановка, аналогичная подстановке (7.9):

$$
\varphi(t)=\operatorname{erf}(t)+e^{-\left(\alpha^{2}-1\right) t^{2}} \sum_{m=0}^{\infty} c_{m} H_{m}(\alpha t) .
$$

В работах $[11],[15],[16],[17]$ доказано сушествование непрерьвного монотонно возрастаюшего нечетного вешественно-аналитического при $t \neq 0$ решения краевой задачи $(8.1),(1.6)$ с единственным вешественным нулем в точке $t=0$, причем

$$
\varphi(t)=\left(a_{1} t\right)^{\frac{1}{2 q+1}}[1+O(|t|)] \text { при } t \rightarrow 0,
$$

где

$$
a_{1}=\frac{4}{\sqrt{\pi}} \int_{0}^{\infty} \varphi(\tau) e^{-\tau^{2}} \tau d \tau>0 .
$$

Как и в случае четного $p$ (см. $\S 6)$, справедлива следующая теорема [15], [16].

Теорема. Если $\varphi(t)$ - решение краевой задачи (8.1), (1.6), то оно непрерывно, функция $\varphi^{2 q+1}(t)$ имеет конечное число нулей $t_{k}$ конечной кратности $\sigma_{k}, k=$ $1, \ldots, l, \sum_{k=1}^{l} \sigma_{k}-$ нечетное число, причем

$$
\varphi(t)=\left[\frac{a_{\sigma_{k}}}{\left(\sigma_{k}\right) !}\right]^{\frac{1}{2 q+1}}\left(t-t_{k}\right)^{\frac{\sigma_{k}}{2 q+1}}\left[1+O\left(\left|t-t_{k}\right|\right)\right] \quad n p u \quad t \rightarrow t_{k},
$$

əде

$$
\frac{2^{\sigma_{k}}}{\sqrt{\pi}} \int_{-\infty}^{\infty} \varphi(\tau)\left(\tau-t_{k}\right)^{n} e^{-\left(t_{k}-\tau\right)^{2}} d \tau= \begin{cases}a_{\sigma_{k}}, & n=\sigma_{k} \\ 0, & n=0,1, \ldots, \sigma_{k}-1\end{cases}
$$

Число перемен знака у функций $\varphi(t)$ и $\varphi^{2 q+1}(t)$ одинаково, нечетно, $\geqslant 3$, не больше l и не меньше $\max _{1 \leqslant k \leqslant l} \sigma_{k}$.

Вопросы. 1) Всегда ли в нуле функиии $\varphi^{2 q+1}(t)$ имеет место перемена знака у решения $\varphi(t)$ ?

2) Будут ли нули функиии $\varphi^{2 q+1}(t)$ простьлми?

Автор благодарит И.Я. Арефьеву, А.В. Воронина и С. В. Козырева за полезные обсуждения и помошь при оформлении рисунков.

\section{СПИСОК ЛИТЕРАТУРЫ}

[1] М. Грин, Дж. Шварц, Э. Виттен. Теория суперструн. Т. I, II. М.: Мир, 1990.

[2] L. Brekke, P. G. O. Freund, M. Olson, E. Witten. Non-Archimedean string dynamics // Nuclear Phys. B. 1988. V. 302. № 3. P. 365-402.

[3] P. H. Frampton, Y. Okada. Effective scalar field theory of $p$-adic string // Phys. Rev. D (3). 1989. V. 37. № 10. P. 3077-3079.

[4] В.С. Владимиров, И.В. Волович, Е.И. Зеленов. p-адический анализ и математическая физика. М.: Наука, 1994.

[5] L. Brekke, P. G.O. Freund. p-Adic numbers in physics // Phys. Rep. 1993. V. 233. № 1. P. $1-66$. 
[6] N. Moeller, B. Zwiebach. Dynamics with infinitely many time derivatives and rolling tachyons // J. High Energy Phys. 2002. № 10. Paper 034; arXiv:hep-th/0207107.

[7] A. Sen. Rolling tachyon // J. High Energy Phys. 2002. № 4. Paper 48; arXiv:hep-th/0203211.

[8] D. Ghoshal, A. Sen. Tachyon condensation and brane descent relations in $p$-adic string theory // Nuclear Phys. B. 2000. V. 584. № 1-2. P. 300-312.

[9] I. Ya. Aref'eva, L. V. Joukovskaya, A. S. Koshelev. Time evolution in superstring field theory on non-BPS brane. I. Rolling tachyon and energy-momentum conservation // J. High Energy Phys. 2003. № 9. Paper 012; arXiv:hep-th/0301137.

[10] I. V. Volovich. p-Adic string // Classical Quantum Gravity. 1987. V. 4. № 4. P. L83-L87.

[11] Ya. Volovich. Numerical study of nonlinear equations with infinite numbers of derivatives // J. Phys. A. 2003. V. 36. № 32. P. 8685-8701; arXiv:math-ph/0301028.

[12] J. A. Minahan. Mode interactions of the tachyon condensate in $p$-adic string theory // J. High Energy Phys. 2001. № 3. Paper 28.

[13] N. Moeller, M. Schnabl. Tachyon condensation in open-closed $p$-adic string theory // J. High Energy Phys. 2004. № 1. Paper 011.

[14] N. Barnaby. Caustic formation in tachyon effective field theories // J. High Energy Phys. 2004. № 7. Paper 025; arXiv:hep-th/0406120.

[15] В.С. Владимиров, Я.И. Волович. О нелинейном уравнении динамики в теории p-адической струны // ТМФ. 2004. Т. 138. № 3. С. 355-368; arXiv:math-ph/0306018.

[16] В. С. Владимиров. Об уравнении $p$-адической открытой струны для скалярного поля тахионов // Изв. РАН. Сер. матем. 2005. Т. 69. № 3. С. 55-80.

[17] Л.В.ЖУуковская. Итерационный метод решения нелинейных интегралшных уравнений, описьвающих роллинговые решения теории струн // ТМФ. (в печати).

[18] А. Ф. Никифоров, В.ББ. Уваров. Специальные функции математической физики. М.: Наука, 1978.

[19] А. П. Прудников, Ю. А. Брычков, О. И. Маричев. Интегралы и ряды. Специальные функции. М.: Физматлит, 1983.

[20] И.С. Градштейн, М.И. Рыжик. Таблицы интегралов, сумм, рядов и произведений. М.: Физматлит, 1963. 